\section{Supersized virtual screening offers potent leads}

Chemical space is vast, and the screening libraries that are used to find starting points for new drugs don't even begin to scratch the surface of what's possible. Computational methods that predict how a given small molecule will bind a target offer the potential to screen large virtual libraries of compounds more cheaply, but any hits still need to be

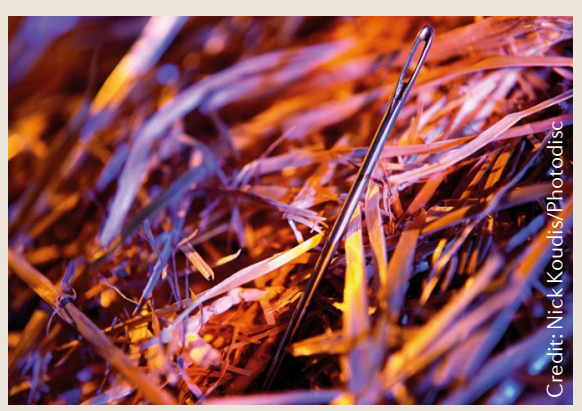
validated in physical screens. Consequently, many virtual screens have been limited to at most a few million commercially available compounds. A recent paper in Nature now showcases the benefits of expanding the size of a virtual screening library into tens of millions of compounds by strategically pre-selecting diverse compounds that can be readily made on demand.

University of California, San Francisco (UCSF)'s Brian Shoichet, University of North Carolina's Bryan Roth, UCSF's John Irwin and colleagues used a structure-based docking approach to screen 99 million and 138 million virtual compounds, respectively, against AmpC $\beta$-lactamase (AmpC) and the $\mathrm{D}_{4}$ dopamine receptor. As all the compounds in these libraries could be readily synthesized, the researchers were able to then quickly order and test a subset of the top hits for activity. This resulted in the identification of 81 new chemotypes, 30 of which had submicromolar activity against their target and one of which had picomolar activity, they report.

"The new work clearly demonstrates the advantages of using ultra-large libraries to discover potent and selective molecules suitable as leads for drug development," wrote the University of Copenhagen's David Gloriam in a linked News and Views article.

"Imagine what that world will look like 5 years from now, when the libraries are trillions of compounds, the scoring functions are quite good and the cost of synthesis is quite low," says Mark Murcko, CSO of Dewpoint Therapeutics and an advocate of computational screening in former roles at Vertex Pharmaceuticals and Relay Therapeutics. "This starts to feel fairly powerful."

But he also notes that these results were possible in part because extensive crystallography work has already been done on $\mathrm{AmpC}$ and the $\mathrm{D}_{4}$ dopamine receptor to inform the docking models. In the case of newer and neglected targets, the results of virtual screens will not be as reliable. "Crystallography could become a bit of a bottleneck if we're not careful," he says.

Asher Mullard

\section{Drug pricing under the spotlight}

In February, US lawmakers grilled pharmaceutical executives from seven firms about the high cost of drugs in the US. While the hearing included considerable political theatre and blame shifting, it also highlighted ways in which the drug pricing landscape could yet change.

Pascal Soriot, CEO of AstraZeneca, proposed that the sector move away from the rebate system, in which health insurance plans and pharmacy benefit managers get rebates from drug companies to offset high list prices. These rebates account on average for "nearly $50 \%$ of [AstraZeneca's] gross revenues in the United States", he told the hearing. If lawmakers eliminated rebates, he said, his firm could cut list prices by an equivalent amount.

Soriot and others also spoke about the promise of value-based pricing, in which prices are linked to pre-specified outcomes. These deals take many forms, but in some payers get reimbursed when a drug doesn't help a patient.
Mark McClellan, of the Duke-Margolis Center for Health Policy, and colleagues reported recently that value-based pricing is increasingly prevalent. The team surveyed 12 biopharmaceutical companies and 13 payer groups about value-based pricing, and found that respondents had already set up 88 and 122 such agreements, respectively, since 2014 . Over $70 \%$ of these deals had not been publicly disclosed, they report in the American Journal of Managed Care.

The 'Netflix' model is also gaining traction in the drug-pricing world, with payers paying a fixed amount for access to unlimited drugs. In one of the largest such deals, the Australian government paid US $\$ 776$ million in 2015 for 5 years of access to hepatitis $C$ drugs. On the basis of drug uptake rates in the first 2 years of this deal, researchers recently calculated a likely per-patient drug price of \$7,400-12,500. This “falls far below" a list price of $\$ 73,000$ (excluding rebates) for hepatitis $\mathrm{C}$ virus drugs in the US at the time of the deal, they wrote in the New England Journal of Medicine.
"Would firms have earned higher revenues through traditional pricing? Possibly, but that was not guaranteed. As many other countries are doing, the government could have restricted patient eligibility in order to control total costs, capping firms' total revenue," they add.

Asher Mullard

\section{Roche, Biogen and AbbVie stake new gene therapy claims}

Big pharmaceutical firms continue to jockey for access to gene therapy expertise, technology and clinical candidates.

In late February, Roche acquired Spark Therapeutics for US $\$ 4.8$ billion. Spark was the first company to secure an FDA approval for a gene therapy product, with the agency's 2017 approval of voretigene neparvovec for the treatment of an inherited form of blindness.

Spark's other clinical-stage gene therapies include SPK-8011, for haemophilia A, and SPK-9001, for haemophilia B. It is also working on other ophthalmological, haematological, lysosomal and neurological gene therapies.

Days later, Biogen announced plans to buy Nightstar Therapeutics for $\$ 800$ million. Nightstar focuses on gene therapies for inherited retinal disorders, and its lead candidate NSR-REP1 is in phase III development for choroideraemia.

AbbVie and Voyager Therapeutics meanwhile expanded a collaboration to develop antibody-encoding gene therapies for neurodegenerative conditions. Under the new deal, the partners will work together on vectorized antibodies directed at pathological species of $\alpha$-synuclein for Parkinson disease and other synucleinopathies.

The firms were already working together on a gene therapy that encodes the production of anti-tau antibodies for Alzheimer disease. Together, these deals could be worth over $\$ 1.5$ billion for Voyager.

Last year, Novartis acquired gene therapy firm AveXis for $\$ 8.7$ billion. GlaxoSmithKline, bucking the trend, transferred its rare disease gene therapy portfolio to Orchard Therapeutics. Their portfolio included Strimvelis, an EMA-approved autologous ex vivo gene therapy for adenosine deaminase severe combined immunodeficiency.

Spark, Nightstar, Voyager, AveXis and other gene therapy pioneers work with adenoassociated virus (AAV)-based candidates. AAV has gained popularity as "the ideal therapeutic vector", wrote University of Massachusetts Medical School's Guangping Gao and colleagues in a recent Review.

Asher Mullard 